\title{
"Carvão+Pedra Calcária": Características Termodinâmicas dos Produtos de Combustão ${ }^{1}$
}

\author{
A.P. SPILIMBERGO, C.A. AUTH, Universidade Regional do Noroeste do Estado \\ do Rio Grande do Sul, UNIJUÍ, Departamento de Física, Estatística e Matemática, \\ Cx.P. 560, 98700-000 Ijuí, RS, Brasil
}

C.A. CASTELLI, Universidade Regional do Noroeste do Estado do Rio Grande do Sul, UNIJUÍ, Programa de Pós Graduação em Modelagem Matemática, Cx.P. 560, 98700-000 Ijuí, RS, Brasil.

\begin{abstract}
Resumo. O presente trabalho destina-se a pesquisar teoricamente os intervalos de temperatura para a captura efetiva dos poluentes do tipo $\mathrm{SO}_{x}$ por pedras calcárias fluidizadas, no processo de combustão do carvão do tipo Antracito, em combustores de leito fluidizado, através de um modelo que considera o equilíbrio químico [1] em um meio reagente composto por 69 substâncias entre gasosas e condensadas. $\mathrm{Na}$ resolução do sistema de equações algébricas não lineares resultante, utiliza-se o método de Newton com algumas modificações, visando garantir a convergência do processo iterativo. O controle da temperatura dos produtos de combustão em uma instalação, para que ocorra a captura efetiva dos poluentes $\mathrm{SO}_{x}$ sem prejuízos para o meio ambiente, é analisado através da injeção de ar e/ou água no meio reagente. As simulações numéricas realizadas mostram que a captura dos poluentes $\mathrm{SO}_{x}$ através da injeção de água nos produtos de combustão só se realiza se houver no meio reagente excesso de oxidante, além disso, se no meio reagente for injetado apenas ar a captura ocorre com eficácia.
\end{abstract}

\section{Introdução}

A matriz energética mundial atual está baseada no petróleo e no gás natural, porém seus recursos são limitados [8]. Por outro lado, a disponibilidade de grandes jazidas de carvão mineral e o baixo custo do carvão vegetal fazem com que, nos países desenvolvidos, pesquisas sobre a possibilidade de utilizar mais amplamente este combustível tenham recebido especial atenção. O Brasil também tem acompanhado esta tendência e já começam a surgir algumas pesquisas sobre a utilização desse combustível.

O carvão utilizado em uma instalação, como o combustor de leito fluidizado circulante, como mostra a Fig. 1 [4], é composto por várias substâncias, sendo uma delas o enxofre (S) e portanto, como conseqüência, nos produtos resultantes de sua

\footnotetext{
${ }^{1}$ Este trabalho foi realizado com o apoio da FAPERGS
} 
combustão, formam-se substâncias altamente poluentes como $\mathrm{SO}, \mathrm{SO}_{2}$ e $\mathrm{SO}_{3}$ que são representados de uma forma geral por $\mathrm{SO}_{x}$.

Estratégias de contenção dos poluentes $\mathrm{SO}_{x}$ tem sido bem sucedidas com a utilização de partículas de pedra calcária $\mathrm{CaCO}_{3} *$ (* - símbolo de substância condensada) lançadas para queimar junto ao carvão no meio reagente, no processo de combustão.

A captura dos poluentes $\mathrm{SO}_{x}$ ocorre em duas etapas. Inicialmente, na zona de

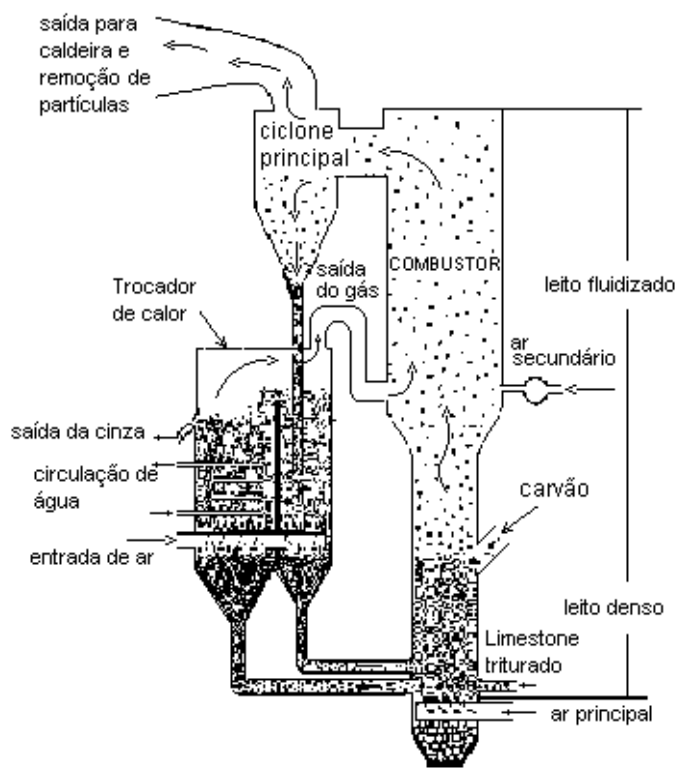

Figura 1: Esquema de um combustor de leito fluidizado circulante

combustão, as partículas de pedras calcárias decompõem-se pela reação: $\mathrm{CaCO}_{3}{ }^{*} \rightarrow$ $\mathrm{CaO}^{*}+\mathrm{CO}_{2}$, com a formação de poros. Logo após as substâncias poluentes $\mathrm{SO}_{x}$, formadas nos produtos de combustão, penetram nos poros e reagem com o óxido de cálcio $\left(\mathrm{CaO}^{*}\right)$, formando com ele o sulfato de cálcio $\left(\mathrm{CaSO}_{4}{ }^{*}\right)$, que é uma substância sólida e não poluente, que pode facilmente ser removida do combustor junto com a cinza, pelas reações:

$$
\mathrm{CaO} *+\mathrm{SO}_{2}+\frac{1}{2} \mathrm{O}_{2} \rightarrow \mathrm{CaSO}_{4} *, \mathrm{CaO}^{*}+\mathrm{SO}+\mathrm{O}_{2} \rightarrow \mathrm{CaSO}_{4} * \mathrm{e} \mathrm{CaO}^{*}+\mathrm{SO}_{3} \rightarrow \mathrm{CaSO}_{4} *
$$

Pesquisar teoricamente os intervalos de captura efetiva dos poluentes $\mathrm{SO}_{x}$ por partículas de pedras calcárias, através de um modelo que considera o meio reagente no estado de equilíbrio químico, é o objetivo deste trabalho. Para isso, no modelo de Alemassov et al. [1], foi introduzido o método das "Grandes Moléculas" [9], para calcular com segurança meios reagentes com variedade expressiva de substâncias condensadas.

Este modelo supõe que existe um meio reagente cujas substâncias que o compõe (essas substâncias sâo átomos e moléculas/radicais) estão em equilíbrio químico entre si e com a temperatura $(T[\mathrm{~K}])$ e a pressão $(P[\mathrm{~Pa}])$. Além disso supõe também 
que para cada substância (átomo ou molécula/radical) a entalpia e a entropia no estado padronizado $\left(\mathrm{H}_{q}=[\mathrm{kJ} / \mathrm{kmol}]\right.$ e $\left.\mathrm{S}_{q}=[\mathrm{kJ} /(\mathrm{kmol} . \mathrm{K})]\right)$, são funções da temperatura e que para cada substância e para a mistura total são válidas as equações de estado de um gás ideal. O meio reagente equilibrado é descrito (se o valor da temperatura é desconhecido) pelo sistema de equações a seguir.

$$
\begin{array}{ll}
\prod_{i=1}^{n} P_{i}^{a_{i j}} / P_{j}=K_{j(P)}, & j=1, \ldots, m \\
\sum_{j=1}^{m} a_{i j} \cdot P_{j}+P_{i}=M_{p} \cdot b_{i p}, & i=1, \ldots, n \\
\sum_{q=1}^{m+n} P_{q}=P & \\
I_{p}-\sum_{q=1}^{m+n}\left(H_{q} \cdot r_{q}\right) / \sum_{q=1}^{m+n}\left(r_{q} \cdot \mu_{q}\right)
\end{array}
$$

onde $P_{i}$ e $P_{j}$ são as pressões parciais do átomo $i$ e molécula/radical $j ; a_{i j}$ é a quantidade do átomo $i$ em uma molécula/radical $j ; K_{j(P)}$ é a constante de dissociação pela pressão; $b_{i p}$ é a quantidade do átomo $i$ na fórmula condicional do bipropelente; $M_{p}$ é a constante de proporcionlidade que assegura a igualdade: $P_{q}=n_{q}\left(n_{q}\right.$ é a quantidade de moles da $q$-ésima substância nos produtos de combustão); $I_{p}$ é a entalpia mássica do bipropelente $[\mathrm{kJ} / \mathrm{kg}] ; \mu_{q}$ e $r_{q}$ são respectivamente massa molecular e fração mássica da $q$-ésima substância nos produtos de combustão.

As relações (1.1)-(1.4) fornecem um volumoso sistema de equações algébricas não lineares, e para sua resolução utiliza-se o método de Newton com algumas modificações que visam garantir a convergência do processo iterativo. Entre elas podemos destacar: escrita das equações na forma logarítmica, teste para detectar o aparecimento de substância condensada, realização dos cálculos com precisão dupla para tentar evitar singularidades e estimativa apropriada das aproximações iniciais para as raízes. O modelo Alemassov et al. [1] se encontra mais detalhadamente em Spilimbergo et al. [10]. A seguir serão mostradas com mais detalhes duas das medidas utilizadas para evitar a divergência.

Para excluir a obtenção de valores negativos nas pressões parciais, no andamento das iterações, nas equações (1.1)-(1.3) é realizada uma troca de variáveis:

$$
\begin{array}{ll}
\gamma_{s}=\ln P_{j} & s=j=1, \ldots, m \\
\gamma_{s}=\ln P_{i} & s=i+m ; i=1, \ldots, n \\
\gamma_{s}=\ln M_{p} & s=m+n+1,
\end{array}
$$

então as equações (1.1)-(1.3) são apresentadas na forma a seguir.

$$
F_{s} \equiv \gamma_{s}-\sum_{i=1}^{n} a_{i s} \cdot \gamma_{(i+m)}+\ln K_{s}=0 \quad s=1, \ldots, m
$$




$$
\begin{array}{cl}
F_{s} \equiv \ln \left(\sum_{j=1}^{m} a_{(s-m) j} \cdot e^{\gamma_{j}}\right)-\gamma_{s}-\ln b_{(s-m) p}=0 & s=(m+1), \ldots,(m+n) \\
F_{s} \equiv \ln \left(\sum_{q=1}^{m+n} e^{\gamma_{q}}\right)-\ln P=0 & s=m+n+1 .
\end{array}
$$

Aplicando o esquema do método de Newton às equações (1.8)-(1.10), obtém-se:

$$
\begin{gathered}
\sum_{v}\left(\frac{\partial F_{s}}{\partial \gamma_{v}}\right)_{p} \cdot \Delta \gamma_{v}=-\delta_{s}^{(p)} \quad v, s=1, \ldots, m+n+1 \\
\gamma_{s}^{(p+1)}=\gamma_{s}^{(p)}+\triangle \gamma_{s},
\end{gathered}
$$

onde: $\triangle \gamma_{v}$ é o incremento; $\delta_{s}^{(p)} \equiv F_{s}^{(p)}$ e $p$ é o número de iterações.

Determinando as derivadas parciais indicadas em (1.11) e fazendo as respectivas substituições obtém-se:

$$
\begin{gathered}
\triangle \gamma_{s}-\sum_{i=1}^{n} a_{i s} \cdot \triangle \gamma_{i+m}=-\delta_{s} \quad s=1, \ldots, m \\
\sum_{j=1}^{m} a_{(s-m) j} P_{j} \triangle \gamma_{j}+P_{s} \Delta \gamma_{s}-B_{s} \triangle \gamma_{(m+n+1)}=-\delta_{s} B_{s} \quad s=(m+1), \ldots,(m+n) \\
\sum_{q=1}^{m+n} P_{q} \cdot \Delta \gamma_{q}=-\delta_{(m+n+1)} \cdot \sum_{q=1}^{m+n} P_{q},
\end{gathered}
$$

onde $B_{s}=\sum a_{(s-m) j} P_{j}+P_{s}$. Este sistema de equações é aplicado em cada interação do método de Newton. Para calcular $\gamma_{s}^{(p+1)}$, é utilizada a fórmula modificada:

$$
\gamma_{s}^{(p+1)}=\gamma_{s}^{(p)}+\xi^{(p)} \triangle \gamma_{s}
$$

onde $\xi^{(p)}$ é o coeficiente do passo de iteração. No trabalho esse coeficiente é determinado de acordo com o que segue. Seja $N_{p}$ o número de iterações e seja $\triangle=\sum\left|\delta_{i}\right|+\sum\left|\delta_{j}\right|+\left|\delta_{c}\right|$, então:

1) se $N_{p}<15$ então $\xi=0,3 / \triangle$

2) se $\xi<0,1$ e $N_{p}>5$ então $\xi=0,1$;

3) se $N_{p}>15$ então $\xi=\left(N_{p} / 15\right)-0,9$;

4) se $\xi>1$ então $\xi=1$;

5) se $N_{p}<10$ e $\xi>0,6$ então $\xi=0,6$.

A precisão é atingida quando $\max \left(\triangle \gamma_{s}\right) \leq 0,0001$.

As aproximações iniciais das raízes do sistema são escolhidas da seguinte forma:

$$
P_{i}^{o}=P_{j}^{o}=P /(m+n) \quad \text { e } \quad M_{p}^{o}=20 P / \mu_{p},
$$

onde $\mu_{p}$ é a massa molecular do bipropelente.

\section{Complementação da Base de Dados}

Para a realização de cálculos através do modelo de equilíbrio químico [1] é utilizado o aplicativo ADTIJUI [2], que está ligado a uma base de dados que contém informações sobre aproximadamente 200 substâncias gasosas e 50 substâncias condensadas, mas para meios reagentes gerados pelo carvão, a base de dados do aplicativo 
ADTIJUI é insuficiente. Portanto foi realizada a sua complementação utilizando os dados de Glushko et al. [3] (Base EVITA), que contém informações sobre um número maior de substâncias.

As informações constantes na base EVITA estão ligadas relativamente a temperatura de $298 \mathrm{~K}$ e são determinadas pelos polinômios de aproximação indicados em (2.1) e (2.2), onde $x=0,0001 T$. As informações constantes em ADTIJUI estão ligadas a temperatura de $293 \mathrm{~K}$ e são determinadas pelos polinômios de aproximação indicados em (2.3) e (2.4), onde $x=0,001 T$ e $w=7$ para substâncias gasosas ou $w=3$ para substâncias condensadas.

$$
\begin{gathered}
H_{q}=\left(\Delta H_{f q}\right)_{298,15}-\left[H_{298,15}^{o}-H_{0}^{o}\right]_{q}+ \\
+10^{-4}\left[-2 a_{2 q} x^{-1}-a_{-1 q}+a_{L q} x+a_{1 q} x^{2}+2 a_{2 q} x^{3}+3 a_{3 q} x^{4}\right] \\
S_{q}=-a_{-2 q} x^{-2}+a_{L q} \ln x+a_{0 q}+a_{L q}+2 a_{1 q} x+3 a_{2 q} x^{2}+4 a_{3 q} x^{3} \\
H_{q}=A_{I_{q}}+\sum_{v=1}^{w} a_{q_{v}} \cdot x^{v} \\
S_{q}=A_{S_{q}}+10^{-3} \cdot a_{q 1} \cdot \ln x+10^{-3} \sum_{v=2}^{w} \frac{v}{(v-1)} \cdot a_{q_{v}} \cdot x^{v-1}
\end{gathered}
$$

Portanto para que a base do aplicativo ADTIJUI fosse complementada com algumas substâncias da base EVITA, se fez necessário realizar uma reaproximação, de modo que os dados das duas bases se tornassem compatíveis, e esta reaproximação foi realizada através do aplicativo auxiliar APRO [2], cujo o algoritmo inclui as etapas a seguir.

1) Leitura dos coeficientes da q-ésima substância da base EVITA: $a_{0}, a_{L}, a_{-2}, a_{-1}$, $a_{1}, a_{2}, a_{3}$.

2) Cálculo do parâmetro para adaptar a entalpia na escala da base de dados de ADTIJUI: $\Delta E s=F_{2}-F_{1}$, onde

$$
F_{z}=10^{-4}\left[-2 a_{2} x^{-1}-a_{-1}+a_{L} x+a_{1} x^{2}+2 a_{2} x^{3}+3 a_{3} x^{4}\right],
$$

com $z=1$ e $2 ; x_{1}=293 / 10000$ e $x_{2}=298 / 10000$.

3) Cálculo de $\tilde{H}_{k}$ e $\tilde{S}_{k}$, para várias temperaturas $T_{k}$, através dos polinômios de aproximação (2.1) e (2.2), alterando a escala da entalpia $\left(\tilde{H}_{k}=H_{k}+\Delta E s\right)$ e formando a matriz $(\mathrm{A}) \Rightarrow T_{k}, \tilde{H}_{k}, \tilde{S}_{k}\left(k=1, \ldots, k_{T}\right)$.

4) Cálculo dos coeficientes dos polinômios (2.3) e (2.4) pelo método de Chebyshev [7], utilizando a matriz (A) onde para substâncias gasosas os coeficientes são: $A_{I}$, $A_{S}, a_{1}, a_{2}, a_{3}, a_{4}, a_{5}, a_{6}, a_{7}$ e para substâncias condensadas são: $A_{I}, A_{S}, a_{1}, a_{2}$, $a_{3}$.

5) Cálculo de $\bar{C}_{p k}$ e também de $\bar{H}_{k}$ e $\bar{S}_{k}$ através dos polinômios de aproximação (2.3) e (2.4), formando a matriz $(\mathrm{B}) \Rightarrow \bar{C}_{p k}, \bar{H}_{k}, \bar{S}_{k}$, com $k=1, \ldots, k_{T}$.

6) Cálculo dos erros de reaproximação:

$$
\varepsilon_{H}=\max a b s\left[\left(\tilde{H}_{k}-\bar{H}_{k}\right) / \bar{C}_{p k}\right] \text { e } \varepsilon_{S}=\max a b s\left[\left(\left(\tilde{S}_{k}-\bar{S}_{k}\right) \cdot T_{k}\right) / \bar{C}_{p k}\right] \text {. }
$$


Quando os erros $\varepsilon_{H}$ e $\varepsilon_{S}$ são admissíveis (da ordem de $1 \mathrm{~K}$ ) os coeficientes calculados no item 4 são incluídos na base de dados do aplicativo ADTIJUI. As grandezas $\varepsilon_{H}$ e $\varepsilon_{S}$ refletem os erros da temperatura dos produtos de combustão provocados pelos erros de reaproximação das propriedades termodinâmicas de cada substância. Por exemplo, o valor $\varepsilon_{H}=0,1$ significa que, usando os dados da reaproximação, a temperatura desta substância como função da entalpia, será calculada com um erro de $0,1 \mathrm{~K}$.

\section{Simulações Numéricas}

$\mathrm{Na}$ literatura encontram-se alguns resultados de pesquisas teóricas sobre a captura dos poluentes $\mathrm{SO}_{x}$, para meios reagentes bastantes simples. Um exemplo disso, mostrado por Lyngfelt e Leckner [5], considera o meio reagente composto pelas substâncias gasosas: $\mathrm{O}_{2}, \mathrm{SO}_{2}, \mathrm{CO}, \mathrm{CO}_{2}, \mathrm{~N}_{2}$ e pelas substâncias condensadas: $\mathrm{CaO}^{*}$, $\mathrm{CaSO}_{4} *$ e CaS*. Não levam em conta, entre outros fatores, a complexidade da composição do carvão e a presença nos produtos de combustão de outras espécies condensadas além de substâncias calcinadas. Para este trabalho o carvão pesquisado foi do tipo Antracito e a sua composição foi dada em Litt et al. [4] e está apresentada na Tabela I.

Tabela I - Composição (\% mássico) e poder calorífico alto $(\Delta \mathrm{Ha})$ do Antracito

\begin{tabular}{|c|c|c|c|c|c|c|c|c|}
\hline Carbono & $\mathrm{H}$ & $\mathrm{O}$ & $\mathrm{S}$ & $\mathrm{N}$ & $\mathrm{Cl}$ & Umidade & Cinza & $\Delta \mathrm{Ha}(\mathrm{Btu} / \mathrm{lb})$ \\
\hline 71,88 & 5,09 & 8,55 & 2,16 & 1,72 & 0,07 & 10,22 & 10,53 & 12,681 \\
\hline
\end{tabular}

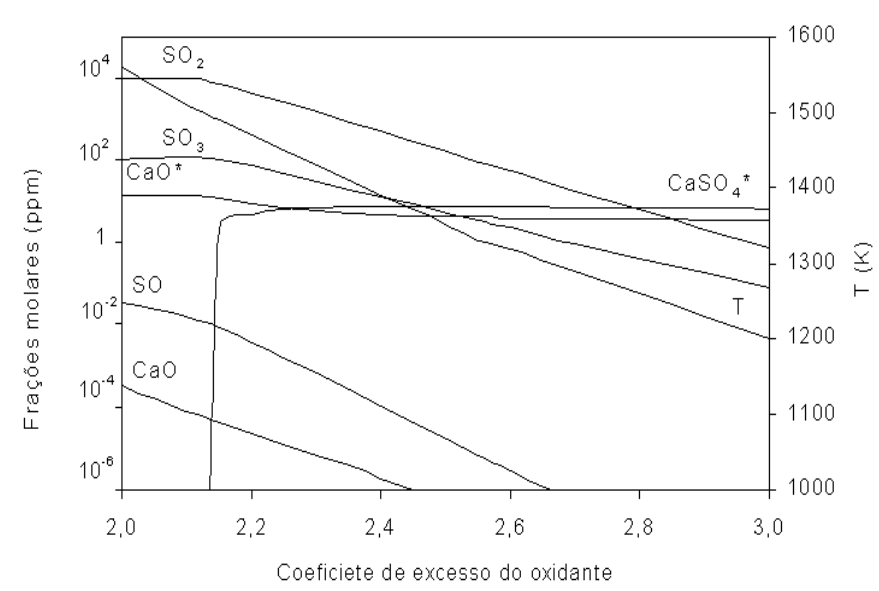

Figura 2: Influência do aumento de $\alpha_{o x}^{f}$ no processo de captura de $\mathrm{SO}_{x}$

Acrescentando ao combustível partículas de pedras calcárias $\left(\mathrm{CaCO}_{3} *\right)$, considerando a relação entre os átomos de cálcio e de enxofre: $\mathrm{Ca} / \mathrm{S}=1,5$ e considerando também, de acordo com W. J. Peet e V. Kannan [6], que a cinza é composta por $50 \%$ de $\mathrm{Al}_{2} \mathrm{O}_{3} *$ e $50 \% \mathrm{de}_{\mathrm{SiO}_{2}}{ }^{*}$, facilmente se obtém a fórmula condicional 
do "Antracito + Pedras Calcárias":

$$
\left[C_{5,160} H_{5,368} N_{0,110} C l_{0,002} S_{0,058} O_{1,317} \mathrm{Ca}_{0,088} S i_{0,074} A l_{0,088}\right]
$$

ou seja, sua composição é bastante complexa. A entalpia foi determinada de acordo com o descrito em Spilimbergo et al [9], e resultou em $I_{c}=-2971 \mathrm{~kJ} / \mathrm{kg}$. Os cálculos foram realizados para $P=10^{5} \mathrm{~Pa}, \alpha_{o x}=0,1 \ldots 3,0$ e o meio reagente foi composto pelas substâncias: $\mathrm{H}, \mathrm{O}, \mathrm{N}, \mathrm{C}, \mathrm{Cl}, \mathrm{S}, \mathrm{Si}, \mathrm{Ca}, \mathrm{Al}, \mathrm{HCl}, \mathrm{HO}_{2}, \mathrm{HS}, \mathrm{H}_{2}, \mathrm{H}_{2} \mathrm{O}, \mathrm{OH}, \mathrm{O}_{2}$, $\mathrm{NO}, \mathrm{NO}_{2}, \mathrm{NH}, \mathrm{NH}_{2}, \mathrm{NH}_{3}, \mathrm{NS}, \mathrm{N}_{2}, \mathrm{CO}, \mathrm{CH}_{4}, \mathrm{CO}_{2}, \mathrm{CH}_{2}, \mathrm{CH}_{3}, \mathrm{CH}, \mathrm{CN}, \mathrm{CS}, \mathrm{CS}_{2}$, $\mathrm{CCl}, \mathrm{CCl}_{2}, \mathrm{CCl}_{3}, \mathrm{CCl}_{4}, \mathrm{ClO}, \mathrm{ClO}_{2}, \mathrm{Cl}_{2}, \mathrm{Cl}_{2} \mathrm{O}, \mathrm{CaO}, \mathrm{SO}, \mathrm{SO}_{2}, \mathrm{SO}_{3}, \mathrm{SiO}_{2}, \mathrm{SiH}$, $\mathrm{SiC}, \mathrm{SiC}_{2}, \mathrm{SiO}, \mathrm{SiCl}_{2}, \mathrm{SiN}, \mathrm{SiCl}, \mathrm{SiCl}_{3}, \mathrm{SiCl}_{4}, \mathrm{Si}_{2} \mathrm{C}, \mathrm{AlH}, \mathrm{AlO}, \mathrm{AlO}_{2}, \mathrm{AlCl}, \mathrm{AlCl}_{3}$, $\mathrm{Al}_{2} \mathrm{O}_{2}, \mathrm{Al}_{2} \mathrm{O}, \mathrm{C}^{*}, \mathrm{CaSO}_{4} *, \mathrm{CaO}^{*}, \mathrm{SiO}_{2} *, \mathrm{Al}_{2} \mathrm{O}_{3} *, \mathrm{CaCO}_{3} *$ e $\mathrm{Ca}^{*}$.

As pesquisas foram realizadas para coeficientes de excesso do oxidante $\left(\alpha_{o x}\right)$ que correspondem as temperaturas de captura dos poluentes $\mathrm{SO}_{x}$ por pedras calcárias $(T=1100, . ., 1600 \mathrm{~K})$.

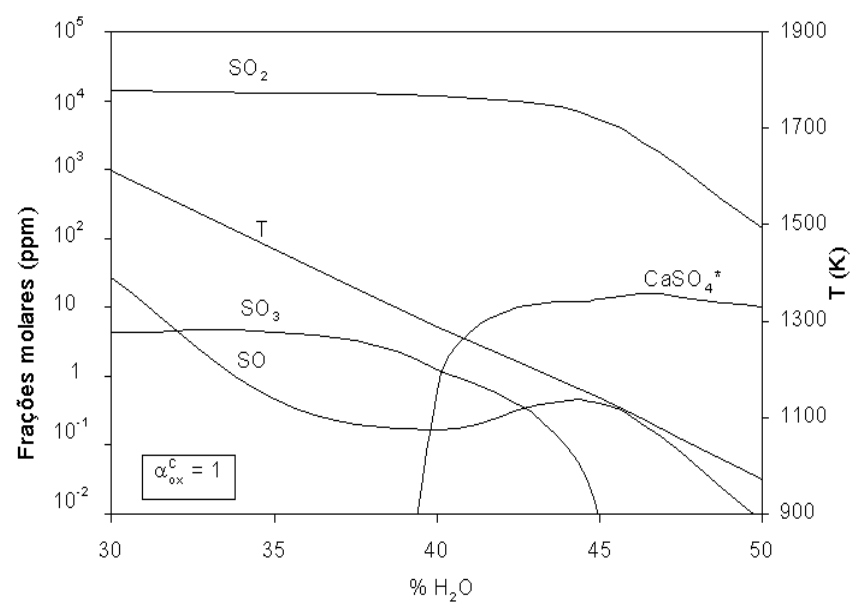

Figura 3: Influência da $\theta_{a g}$ na captura de $\mathrm{SO}_{x}$ por pedras calcárias $\left(\alpha_{o x}^{c}=1\right)$

No combustor (Fig. 1) ocorre, logo após a zona de combustão (onde $\alpha_{o x}^{c} \approx 1$ ) a admissão de ar secundário $\left(\alpha_{o x}^{f}>1\right)$ e a sua influência na captura dos poluentes $\mathrm{SO}_{x}$ está mostrada na Fig. 2. Verifica-se que para $\alpha_{o x}^{f}>2,2$ (o que corresponde a $T \leq 1450 \mathrm{~K})$ o óxido de cálcio $\left(\mathrm{CaO}^{*}\right)$ reage com os produtos de combustão $\mathrm{SO}_{x}$ e transforma-se em sulfato de cálcio, confirmando assim, a captura eficaz desses poluentes, por pedras calcárias, neste tipo de instalação.

As figuras de 3 e 4 mostram a influência da injeção de $\mathrm{H}_{2} \mathrm{O}$ no meio reagente na captura dos poluentes $\mathrm{SO}_{x}$ por pedras calcárias em duas situações: $\alpha_{o x}^{c}=1 \mathrm{e}$ $\alpha_{o x}^{c}=1,25$, sem admissão de ar secundário.

Quando $\alpha_{o x}^{c}=1$ (Fig. 3), pesquisou-se taxas de injeção de água $\left(\theta_{a g}\right)$ de $30 \%$ a $50 \%$ para a temperatura dos produtos de combustão variar no intervalo de 1000 a $1600 \mathrm{~K}$. Pode-se observar que das substâncias poluentes, formadas a partir do enxofre, a de maior concentração é o $\mathrm{SO}_{2}$. Para $\theta_{a g}=30 \%, \ldots, 42 \%$ o $\mathrm{SO}_{2}$ não é 
capturado, apesar das temperaturas serem favoráveis a isto. Teoricamente a captura do $\mathrm{SO}_{2}$ começa a ser realizada para $\theta_{a g}>45 \%$, mas neste caso $T<1100 \mathrm{~K}$, e por serem as velocidades das reações muito lentas [11] a captura não ocorre, portanto $\operatorname{com} \alpha_{o x}^{c}=1$ a iniecão de água impossibilita a captura do $\mathrm{SO}_{\text {? }}$.

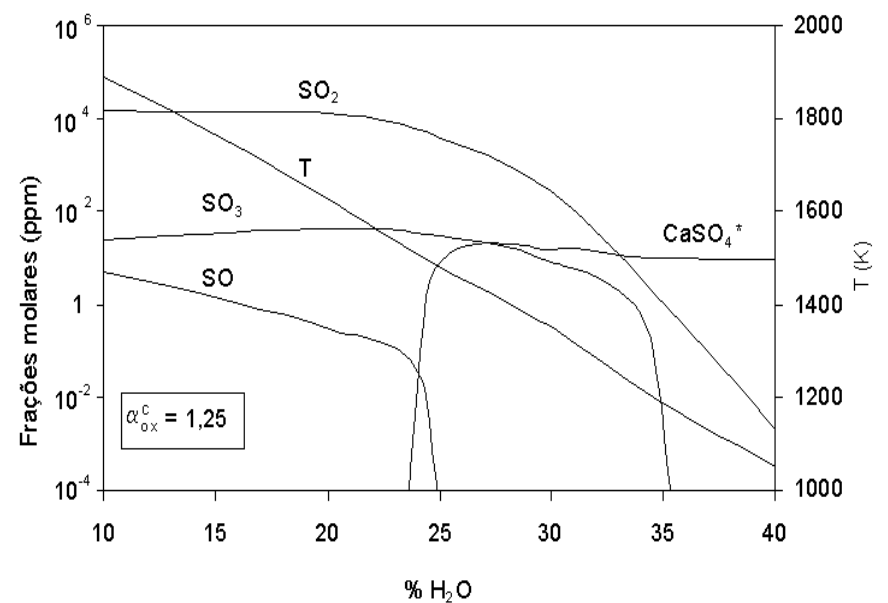

Figura 4: Influência da $\theta_{a g}$ na captura de $\mathrm{SO}_{x}$ por pedras calcárias $\left(\alpha_{o x}^{c}=1,25\right)$

A Fig. 4 mostra a influência da $\theta_{a g}$ na captura dos poluentes $\mathrm{SO}_{x}$ quando $\alpha_{o x}^{c}=1,25$ e verifica-se que para $\theta_{a g} \approx 24 \%$, o que corresponde a $T \approx 1500 \mathrm{~K}$, as substâncias $\mathrm{SO}$ e $\mathrm{SO}_{3}$ já estão totalmente capturadas e começa a redução do $\mathrm{SO}_{2}$. É conhecido que o nível admissível de emissão de $\mathrm{SO}_{2}$ no meio ambiente é em torno de 100ppm, o que corresponde a $T \approx 1300 \mathrm{~K}$ e esta temperatura entra na faixa da captura efetiva. Portanto quando $\alpha_{o x}^{c}=1,25$ a injeção de água no meio reagente realmente contribui para a captura dos poluentes $\mathrm{SO}_{x}$.

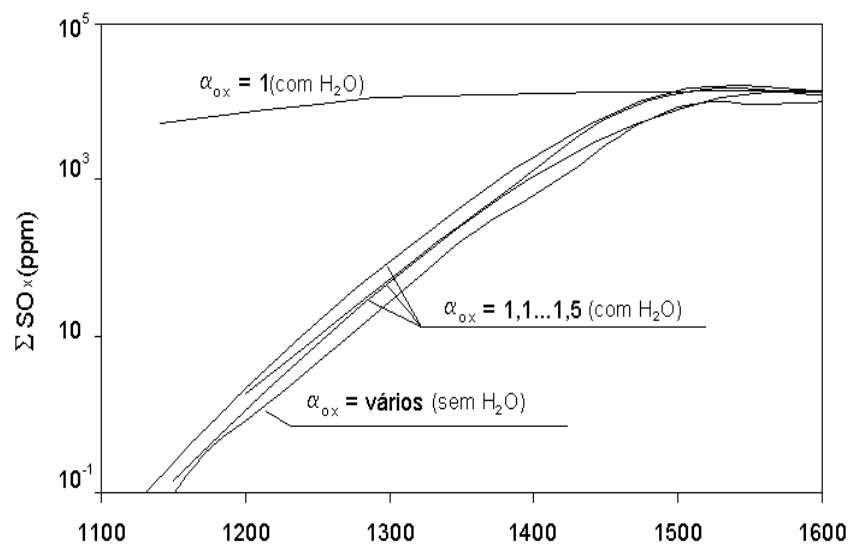

Figura 5: Alteração de $r_{\mathrm{SO}_{2}}=f^{\top}(\stackrel{\mathbb{T}}{T})$ para vários meios reagentes 
Pela análise feita nos últimos dois gráficos, pode-se concluir que havendo a injeção de água na captura dos poluentes $\mathrm{SO}_{x}$, o regime operacional preferível da instalação é com $\alpha_{o x}^{c}$ da ordem de 1,25 .

$\mathrm{Na}$ Fig. 5 são comparadas a capacidade de capturar os poluentes $\mathrm{SO}_{x}$ por pedras calcárias para vários $\alpha_{o x}$, mantendo o intervalo de temperatura dos produtos de combustão variando de 1100 a $1600 \mathrm{~K}$, que é controlado pela injeção de água ou pela admissão de ar meio reagente. O gráfico mostra que as linhas de captura agrupam-se, se no meio reagente existe algum excesso de ar, ou seja $\alpha_{o x}^{c}>1$. Mas quando $\alpha_{o x}^{c}=1$, a captura não se realiza seja qual for a temperatura. Para temperaturas de aproximadamente $1500 \mathrm{~K}$, existe no gráfico um ponto de bifurcação, que é interessante pesquisar futuramente, analisando as equações do modelo de equilíbrio químico.

\title{
4. Conclusões
}

Para o meio reagente "Antracito + Ar" foi pesquisada a capacidade de capturar os poluentes $\mathrm{SO}_{x}$ por pedras calcárias fluidizadas, variando a taxa de injeção de ar secundário e/ou água nos produtos de combustão. As simulações numéricas foram executadas usando o modelo de processos quimicamente equilibrados [1] e o aplicativo ADTIJUI [2]. Em relação aos resultados pode-se fazer as seguintes considerações:

- a captura dos poluentes $\mathrm{SO}_{x}$ pode ser realizada para $T<1550 \mathrm{~K}$, quando $r_{S O_{x}}$ diminui de $10000 \mathrm{ppm}$ até $10 \mathrm{ppm}$;

- para $\alpha_{o x}^{f}=2,0, \ldots, 3,0$ (sem injeção de $\mathrm{H}_{2} \mathrm{O}$ ) no meio reagente pesquisado, o $\mathrm{SO}_{x}$ é capturado com eficácia;

- para o caso da injeção de $\mathrm{H}_{2} \mathrm{O}$, a captura de $\mathrm{SO}_{x}$ não se realiza quando $\alpha_{o x}^{c}=1$, independente da taxa de injeção;

- para $\alpha_{o x}^{c}>1$ a captura do $\mathrm{SO}_{x}$ é considerável quando a taxa de injeção de $\mathrm{H}_{2} \mathrm{O}$ assegura a faixa de temperatura de 1100 a $1300 \mathrm{~K}$.

\begin{abstract}
The present work is to consider a theoretical study of the temperature intervals for the effective capture of pollutants type $\mathrm{SO}_{x}$ by liquefied calcareous stones in the combustion process of the coal type Anthracite. For the resolution of the resulting non-linear algebraic equations system the Newton Method with a few modifications is used in order to guarantee the convergence of the iterative process. The temperature control of the combustion products in an installation, so that the effective capture of the $\mathrm{SO}_{x}$ pollutants occurs without damage to the environment, is analyzed through the injection of air and/or water in the reagent environment. Numerical simulations show that the capture of the $\mathrm{SO}_{x}$ pollutants through the injection of water in the combustion products happens only if there are excess of oxidizer in the reagent environment. Furthermore, if only air is injected in the reagent enviroment, the capture occurs effectively.
\end{abstract}




\section{Referências}

[1] V.E. Alemassov, A.F. Dregalin and A.P. Tishin, "Teoria dos Propulsores", Mashinostroenie, Moscou, 1980.

[2] C.A. Castelli, "Pesquisa Numérica das Características Termodinâmicas e Termofísicas dos Produtos de Combustão de Diferentes Espécies de Carvão", Dissertação de Mestrado, Ijuí, RS, 2000.

[3] Glushko et al., "Guia das Propriedades Termodinâmicas e Termofísicas de Substâncias Individuais", VINITI, Vol. 1 a 12, Moscou, 1980.

[4] R.D. Litt, H. Nack, B.C. Kim and R. Tumolo, Cofiring coal with waste materials, em "Proceedings of First Annual Pittsburgh Coal Conference", pp. 453-459, Pittsburgh, Pennsylvania, 1984.

[5] A. Lyngfelt and B. Leckner, Model of sulphur capture in fluidised-bed boilers under conditions changing between oxidising and reducing, em "Chemical Engineering Science", 48, pp. 31-1141, Great Britain, 1993.

[6] W.J. Peet and V. Kannan, Utility boiler desingn for low volatile coals, em "Coal combustion: Science and Tecnology of Industrial and Utility Applications", pp. 623-632, Ed. Hemisphere Publishing.

[7] W.H. Pess et al., "Numerical Recipes in C", Cambride University Press, New York, 1988.

[8] P.C.C. Pinheiro, Recursos naturis de energia, em "Proceedings of $6^{\text {th }}$ Latin Americn Congress of Heat and Mass Transfer", Vol. I, pp. 189-194, Florinópolis, SC, 1996.

[9] A.P. Spilimbergo, C.J. Auth and R.L. Iskhakova, Método do meio local reagente e grandes moléculas aplicando sistemas quimicamente equilibrado, em "Proceedings of 7th LATCYM, Vol. I, pp. 87-92, Salta, Argentina", 1998.

[10] A.P. Spilimbergo, C.A. Castelli and C.J. Auth, Simulação numérica das propriedades dos produtos de combustão de diferentes espécies de carvão, em "Proceedings of XX Computational Methods in Engineering' 99", CD-ROOM, Escola Politécnica da USP, São Paulo, SP, 1999.

[11] M.R. Stouffer and H. Yoon, An investigation of $\mathrm{CaO}$ sulfation mechanisms in boiler sorbent injection, AIChE Journal 35 (1989), 1253-262. 